\title{
Hedgehog pathway inhibitor-4 suppresses malignant properties of chondrosarcoma cells by disturbing tumor ciliogenesis
}

\author{
WEI XIANG ${ }^{1}$, TING JIANG ${ }^{2}$, FENGJING GUO ${ }^{1}$, CHEN GONG $^{3}$, KAIXIANG YANG ${ }^{1}$, \\ YINGXING WU ${ }^{1}$, XIN HUANG ${ }^{1}$, WEITING $\mathrm{CHENG}^{4}$ and $\mathrm{KAI} \mathrm{XU}^{1}$ \\ Departments of ${ }^{1}$ Orthopedics, ${ }^{2}$ Rehabilitation and ${ }^{3}$ Oncology, Tongji Hospital, Tongji Medical College, Huazhong University \\ of Science and Technology, Wuhan, Hubei 430030; ${ }^{4}$ Department of Oncology, Wuhan Integrated Traditional Chinese \\ Medicine and Western Medicine Hospital, Wuhan No. 1 Hospital, Wuhan, Hubei 430030, P.R. China
}

Received May 13, 2014; Accepted July 8, 2014

DOI: $10.3892 /$ or.2014.3372

\begin{abstract}
Chondrosarcoma is a type of malignant bone tumor secreting cartilage-like matrix. In clinical treatment, there is no frequently used drug treatment option except for surgical resection. Hedgehog $(\mathrm{HH})$ pathway is a classical signaling pathway that regulates normal cartilage cell development. In order to detect the role that $\mathrm{HH}$ pathway plays in chondrosarcoma, we used immunohistochemistry and found this tumor clearly expressed $\mathrm{HH}$ pathway-related proteins. Treatment with $\mathrm{HH}$ pathway inhibitor-4 (HPI-4) could significantly decrease human chondrosarcoma cell proliferation, invasion and migration ability. Furthermore, HPI-4 could distinctly disturb $\mathrm{HH}$ pathway-mediated ciliogenesis and suppress primary cilia-related protein intraflagellar transport protein IFT88 expression. HH downstream effect molecular GLI2 was restrained to block parathyroid hormone-related protein and affect MAPK/ERK-regulated matrix metalloproteinases (MMP2 and MMP9). These results indicated that activated $\mathrm{HH}$ pathway existed in chondrosarcoma and HPI-4 could be a new therapeutic option specific to chondrosarcoma expressing elevated levels of HH pathway.
\end{abstract}

\section{Introduction}

Chondrosarcoma is the second most common malignant tumor originating from bone tissue. It appears mostly in

Correspondence to: Dr Kai Xu, Department of Orthopedics, Tongji Hospital, Tongji Medical College, Huazhong University of Science and Technology, 1095 Jiefang Avenue, Wuhan, Hubei 430030, P.R. China E-mail: kaixutjh@163.com

Abbreviations: HPI-4, Hedgehog pathway inhibitor-4; Ihh, Indian Hedgehog; PTHrP, parathyroid hormone-related protein; PTH, parathyroid hormone; IFT88, intraflagellar transport protein 88; PTCH1, patched protein 1; Smo, Smoothened

Key words: chondrosarcoma, Hedgehog pathway inhibitor-4, proliferation, invasion, migration, primary cilia 30-60-year-old patients. With complex clinical characteristics, pathological manifestations and various differentiation degrees, chondrosarcoma is not sensitive to either radiotherapy or chemotherapy and surgical resection remains the most common treatment in the clinic (1).

Hedgehog $(\mathrm{HH})$ pathway is a crucial signaling pathway in regulating cell growth and differentiation, particularly in bone and cartilage tissues (2,3). Indian hedgehog (Ihh) ligands first bind to patched protein 1 (PTCH1) receptor and then release the repression of Smoothened (Smo) receptor which initiates the activation of downstream transcription factor GLI family $(2,4)$. This process leads to downstream gene expression and regulation of multifarious physiological functions. Our previous studies showed that an $\mathrm{HH} /$ parathyroid hormone-related protein (PTHrP) negative feedback loop exists to regulate the development of different levels of cartilage cells. Ihh facilitates PTHrP expression to promote the growth plate cell proliferation, inhibition of cell differentiation and maturation. Otherwise, PTHrP reduces Ihh production conversely $(2,3)$. More specifically, $\mathrm{HH}$ pathway controls transcription factor GLI2 to control secretion of PTHrP protein which can promote osteolytic destruction, especially in primary or metastatic bone tumor $(5,6)$. Recently, that abnormal activation of $\mathrm{HH}$ pathway induces carcinogenesis was found in various tumor types, such as rhabdomyosarcoma, breast cancer, gastrointestinal malignant tumor and human renal cell carcinomas (7-11). These studies confirmed that overexpression of GLI transcription factors caused the tumorigenesis by affecting cell growth and differentiation progression. Moreover, the malignant properties of these neoplasms were determined by the differentiation and maturation degree of tumors (7-11). In this study, we found that human chondrosarcoma tissue expressed the seemingly abnormal level of $\mathrm{HH}$ signaling-related proteins, such as Ihh, GLI1, GLI2 and PTHrP. However, to date, there is no evidence to illustrate how $\mathrm{HH}$ signaling pathways affect the growth process of chondrosarcoma and whether targeting the $\mathrm{HH}$ pathway could be an optional therapy to treat this tumor (2). Based on our previous findings, we speculated that the $\mathrm{HH}$ signaling pathway may be a potential antitumor target for drug therapy. To test this hypothesis, we treated human chondrosarcoma SW1353 cells with the HH pathway inhibitor, 
HH pathway inhibitor-4 (HPI-4). HPI-4 is a special type of inhibitor which may affect the downstream of Smo but does not directly act on transcription factor GLIs $(13,14)$.

Primary cilia, as a crucial part of the $\mathrm{HH}$ signaling pathway, are special external cellular organelles. Since they contain various receptor proteins, they are always regarded as important extracellular chemical and physical sensors in regulating the cell growth and development process. Cilia can influence the process of differentiation through regulating cell division cycle. Primary cilia's abnormal occurrence, morphology and function are closely related to tumorigenesis (15-19). We focused on the cilia to study the effects of HPI-4 on chondrosarcoma cells by investigating the effects on tumor malignant characteristics, such as proliferation, invasion and migration ability, so as to determine whether this drug has a negative regulation on human chondrosarcoma. In addition, the potential mechanism of HPI-4 through working on primary cilia to regulate Ihh-PTHrP signaling in vitro was investigated. In conclusion, these results indicated that HPI-4 blocking $\mathrm{HH}$ signaling may be a feasible chemotherapeutic option for human chondrosarcoma treatment.

\section{Materials and methods}

Cells and reagents. The human chondrosarcoma cell line SW1353 was purchased from the Type Culture Collection of the Chinese Academy of Sciences (Shanghai, China). Cells were cultured in Dulbecco's modified Eagle's medium/nutrient mixture F-12 (DMEM/F12) with $10 \%$ fetal bovine serum (FBS), penicillin $(100 \mathrm{U} / \mathrm{ml})$ and streptomycin $(100 \mathrm{U} / \mathrm{ml})$ at $37^{\circ} \mathrm{C}$ with $5 \% \mathrm{CO}_{2}$. HPI-4, also known as Ciliobrevin A, whose molecular formula is $\mathrm{C}_{17} \mathrm{H}_{9} \mathrm{Cl}_{2} \mathrm{~N}_{3} \mathrm{O}_{2}$, was purchased from Sigma-Aldrich (St. Louis, MO, USA). The study was approved by the Ethics Committee of Tongji Medical College, Huazhong University of Science and Technology (Wuhan, Hubei, China).

Cell proliferation-toxicity test. To evaluate the change of cell proliferation ability, we used the 3-(4,5-dimethylthiazol-2-yl)5-(3-carboxymethoxyphenyl)-2-(4-sulfophenyl)-2H-tetrazolium (MTS-8) assay to measure cell proliferation and the toxicity of this drug. The procedure was as follows: 2,000 cells were plated in 96-well plates per well. HPI-4 was added to cells at concentrations of 0,5 and $10 \mu \mathrm{M}$ in $100 \mu \mathrm{l} \mathrm{DMEM} / \mathrm{F} 12$ with $10 \%$ FBS and incubated for $0,1,3,6$ and 9 days. Then, $10 \mu \mathrm{l}$ MST-8 (Beyotime, China) was added to the media in each well and incubated in an environment without light for $90 \mathrm{~min}$. The absorbance value was measured using an enzyme microplate reader at $450 \mathrm{~nm}$ wavelength. The relative viability of cells was expressed by OD value.

Tablet colony formation assay. SW1353 cells were harvested by trypsin enzyme digesting and 200 cells were seeded in a 6-well plate. After cells attached, HPI-4 was added to cells at different concentrations of 0,5 and $10 \mu \mathrm{M}$ in each well. Cells were cultured in DMEM/F12 medium with $10 \%$ FBS and incubated at $37^{\circ} \mathrm{C}$ with $5 \% \mathrm{CO}_{2}$ and fresh medium was changed every 3 days. Four weeks later, cells were fixed with $4 \%$ paraformaldehyde and stained with $0.2 \%$ crystal violet. Finally, images were captured to record the size and number of colonies.
Transwell invasion assay. The invasion assay was conducted using Transwell plates. The filter membrane (pore size, $8 \mu \mathrm{m}$ ) of the Transwell plates was coated with $20 \mathrm{mg}$ Matrigel (BD Biosciences, USA) at $37^{\circ} \mathrm{C}$ for $30 \mathrm{~min}$ and the lower chamber was filled with culture medium DMEM/F12 containing $10 \%$ FBS. SW1353 cells were starved with serum-free DMEM/F12 for $6 \mathrm{~h}$ and then $1 \times 10^{5}$ cells were transferred onto the upper surface of the chamber. HPI-4 was added at different concentrations of 0,5 and $10 \mu \mathrm{M}$. Twenty-four hours later, the culture medium was gently removed and the upper surface Matrigel was wiped by cotton swab. We used $4 \%$ paraformaldehyde to fix the cells that had invaded through and adhered to the lower surface for $15 \mathrm{~min}$, then stained with $0.2 \%$ crystal violet. Ten random fields in each plate were selected and counted using an Olympus microscope.

Wound healing assay. Tumor migratory behavior was imitated with a scratch assay by measuring migration distance. First, SW1353 cells were seeded in 6-well plates at a density of $1 \times 10^{5} /$ well. When they reached nearly confluent monolayer, a single scratch was made vertically through each well by use of a sterile pipette tip. After washing with PBS three times, the cells were incubated with $2 \%$ bovine serum DMEM/F12 and cells were stimulated with different concentrations of HPI-4, and observed under a microscope (10x objective lens) after 0,24 and $48 \mathrm{~h}$. Images were recorded at the same position in order to assess the repair process accurately. Finally, the percentage of cell migration distance was calculated.

Immunohistochemical studies. We collected 10 human chondrosarcoma tissues from surgical resection and these tissues were firstly fixed in $4 \%$ paraformaldehyde. They were then embedded in paraffin and sectioned for immunohistochemical assays. All experimental processes were conducted using standard techniques (12). Ihh (1:100; Santa Cruz Biotechnology, Inc., Santa Cruz, CA, USA), GLI1 (1:400; Epitomics Inc., Burlingame, CA, USA), GLI2 (1:100; Boster, Wuhan, China) and PTHrP (1:100; Santa Cruz Biotechnology, Inc.) were used as markers for activation of $\mathrm{HH}$ signaling pathway. Ki67 staining was used to detect proliferation cells in tissue sections at a dilution of 1:200 (Cell Signaling Technology, USA). All sections were observed under a microscope at x200 magnification.

Immunofluorescence assay. SW1353 cells were cultured on the cover glass and treated with different concentrations of HPI-4. Twenty-four hours later, they were fixed with $4 \%$ paraformaldehyde for $15 \mathrm{~min}$, blocked with 5\% BSA at room temperature for $60 \mathrm{~min}$ and then incubated with acetylated $\alpha$-tubulin antibody (1:400; Abcam, Cambridge, UK) overnight at $4^{\circ} \mathrm{C}$. Subsequently, the SW1353 cells were incubated with Cy3-conjugated goat anti-Mouse IgG secondary antibody (Boster) at room temperature and nuclei were stained with $1 \mu \mathrm{g} / \mu \mathrm{l}$ DAPI. We used PBS to wash cells three times during each step during this process. In the end, images were visualized with fluorescent microscope at x200 magnification.

Quantitative real-time PCR ( $q R T-P C R)$. HH signaling pathway-related gene (GLI-1, PTCH1, Smo, IFT88, PTHrP) expression was measured by RT-PCR. Total RNA was 
extracted from cells with TRIzol reagent after $48 \mathrm{~h}$ incubation with HPI-4 in 6-well plates and then 2-5 $\mu \mathrm{g}$ of total RNA was used to synthesize cDNA with the SuperScript II cDNA synthesis kit (Invitrogen Life Technologies, Carlsbad, CA, USA) according to the manufacturer's instructions. The total PCR system contained cDNA, SYBR-Green, no RNA enzyme water and primers; the primer sequences are listed in Table I.

Western blot analysis. These procedures were based on previously described ones (6). Total cell proteins (45 $\mu \mathrm{g} / \mathrm{lane})$ were loaded and separated by sodium dodecyl sulfate (SDS) polyacrylamide gels, transferred onto PVDF membranes. We used the following primary antibodies: MMP2 $(1: 1,000)$, MMP9 (1:1,000) (both from Cell Signaling Technology), IFT88 (1:1,000; Abgent, USA), CyclinD1 (1:100; Boster) and PTHrP (1:100; Santa Cruz Biotechnology, Inc.); $\beta$-actin (1:400; Boster), along with secondary antibody horseradish peroxidase-labeled goat anti-rabbit and goat anti-mouse IgG (1:5,000; Boster). We used ECL western blotting detection kit (Thermo Fisher Scientific, USA) to detect all the protein bands and were visualized using an enhanced chemiluminescence system (Bio-Rad, Hercules, CA, USA). All values were expressed relative to $\beta$-actin.

Statistical analysis. Each experiment was performed at least three times independently. These data are represented as mean \pm SD. We used the Student's t-test or one-way analysis of variance (ANOVA) to analyze the differences between means, and $\mathrm{P}<0.05$ was considered to indicate a statistically significant difference. All statistical analyses were performed using SPSS 20.0.

\section{Results}

Human chondrosarcoma prominently expresses Ihh-PTHrP signaling-related proteins. Previous studies demonstrated that a variety of tumors exist with abnormal activation of the $\mathrm{HH}$ signaling pathway (7-11). Using immunohistochemistry assay, we detected the expression of the Ihh-PTHrP-related proteins in human chondrosarcoma tissues. These tumor tissues exhibited significantly high level of staining intensity for Ihh, GLI1, GLI2 and PTHrP (Fig. 1B-E) compared with the negative control (Fig. 1A). At the same time, tumor tissues had a considerable percentage of positive Ki67 staining in approximately half of total tumor cells $(44.30 \pm 4.52 \%)$. Obvious expression of Ki67 confirmed that chondrosarcoma had considerable proliferation ability (Fig. 1F). On the other hand, apparent expression of Ihh and PTHrP simultaneously indicated that there appeared no natural negative feedback regulation between Ihh and PTHrP proteins. These results demonstrated that the distinct activation of $\mathrm{HH}$ signaling pathway may have a positively correlated relationship with elevated proliferation ability in chondrosarcoma.

HPI-4 decreases chondrosarcoma SW1353 cell proliferation ability. As shown in Fig. 2, in order to examine the effect of HPI-4 treatment on human chondrosarcoma cell growth, SW1353 cells were stimulated respectively with 5 and $10 \mu \mathrm{M}$ HPI-4 and the changes were explored using MTS- 8 assay. With the progress of time, SW1353 cells cultured with HPI-4
Table I. Quantitative real-time PCR primer sequences.

\begin{tabular}{lc}
\hline Gene & \multicolumn{1}{c}{ Primer sequences } \\
\hline hPTCH1 & F: 5'-GTGGTGTAGAGGCAGGCAT-3' \\
& R: 5'-GTGCTGGTCTCTGGTTACGA-3' \\
hGLI1 & F: 5'-TCAAAGTGGGAGGCACAAAC-3' \\
& R: 5'-ATGGGAAGGAGGAGGACTCA-3' \\
hIFT88 & F: 5'-GTTATGATTGGTGCGTGGAAGT-3' \\
& R: 5'-GGGCTGAGAGATTGGTTGCAG-3' \\
hPTHrP & F:5'-AAGGTGGAGACGTACAAAGAC-3' \\
& R: 5'-CAGAGCGAGTTCGCCGTTT-3' \\
hSmo & F: 5'-GACTTCCTGCGCTGCACTC-3' \\
& R: 5'-AGCCCTCCACGTCCTCGTAC-3' \\
h $\beta$-actin & F: 5'-CATGTACGTTGCTATCCAGGC-3' \\
& R: 5'-CTCCTTAATGTCACGCACGAT3'
\end{tabular}

revealed an obvious reduction in proliferation rate compared with the empty control group (Fig. 2A). In addition, in order to detect the effect of this drug on a single cell to form a tumor mass, we conducted a tablet colony formation assay (Fig. 2B). After 4 weeks of incubation, the empty control group number was $272 \pm 19.8$ per well compared with $5 \mu \mathrm{M}(191.00 \pm 8.49$, $\mathrm{P}<0.05)$ and $10 \mu \mathrm{M}(90.50 \pm 16.26, \mathrm{P}<0.05)$. The average size showed the same trends at different doses (Fig. 2B). These results confirmed that HPI-4 clearly reduced the ability of a single cancer cell to generate a colony by continuous division, including numbers and sizes of colonies (Fig. 3C). Moreover, western blot assay presented a similar conclusion that inhibiting $\mathrm{HH}$ signaling eventually suppressed cell cycle protein CyclinD1 in HPI-4-treated cells along with the higher concentration (Fig. 7B). CyclinD1 is considered a vital symbol of cell cycle progression.

HPI-4 inhibits the invasion and migration ability of human chondrosarcoma cells. Migration and invasion are important indicators to evaluate the degree of tumor malignancy, they determine the severity of the tumor and the prognosis of treatment $(7,8)$. In order to illustrate the influence of HPI-4 compound on tumor migration and invasion ability, we performed the Transwell and cell scratch experiment in vitro. Fig. 3A-D shows that the number of cells penetrating through the Matrigel matrix decreased significantly as the drug concentration increased (the white arrows). Matrigel matrix is regarded as the best component to imitate extracellular matrix. Scratch experiment was used to simulate the migration of tumor cells. The results showed that a higher concentration of HPI-4 observably inhibited tumor cell migration rate as time passed. HPI-4 effectively delayed scratch healing time (Fig. 4A and B). At the same time, we conducted western blot experiment to test the expression of matrix metalloproteinases (MMPs) which reflects tumor cell decomposition of the extracellular matrix ability to promote invasion and migration $(22,23)$. The results demonstrated that after stimulating for 3 days, the expression level of MMP2, MMP9 decreased in HPI-4-treated cells. Moreover, as MMPs upstream signaling 

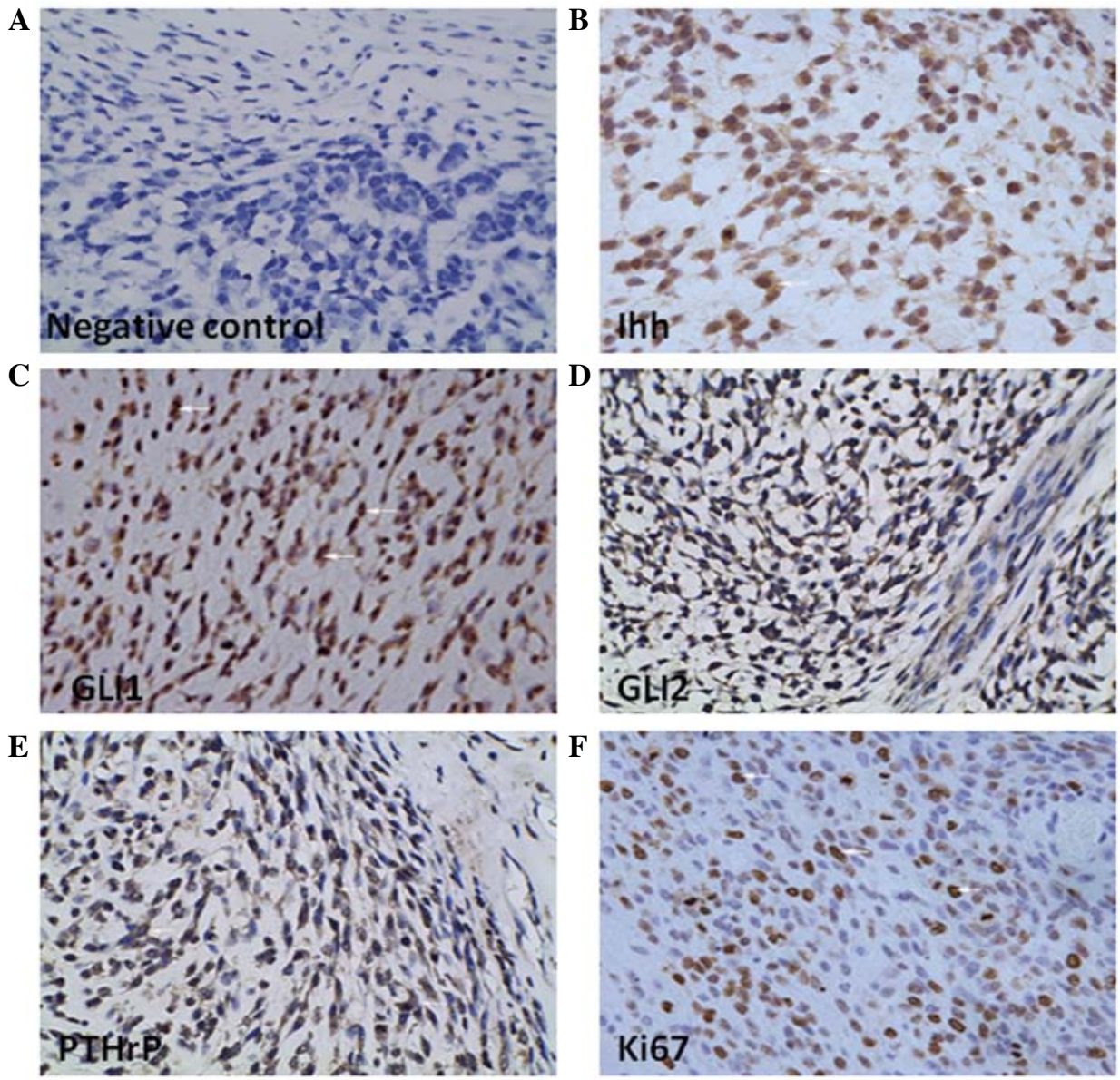

Figure 1. Immunohistochemical staining of HH pathway-related proteins in human chondrosarcoma tissues. (A) Representative staining as negative control without first antibody in human chondrosarcoma tissues. HH signaling pathway target protein (B) Ihh, (C) GLI1 and (D) GLI2 were clearly stained positive. (E) Downstream target protein PTHrP was also stained clear brown and this indicated an activation of PTHrP-regulated proliferation. (F) Nearly half of total tumor cells $(44.30 \pm 4.52 \%)$ presented significantly positive Ki67 expression. Similar to the white arrow, a brown color in chondrosarcoma tissue is defined as a positive staining.

A

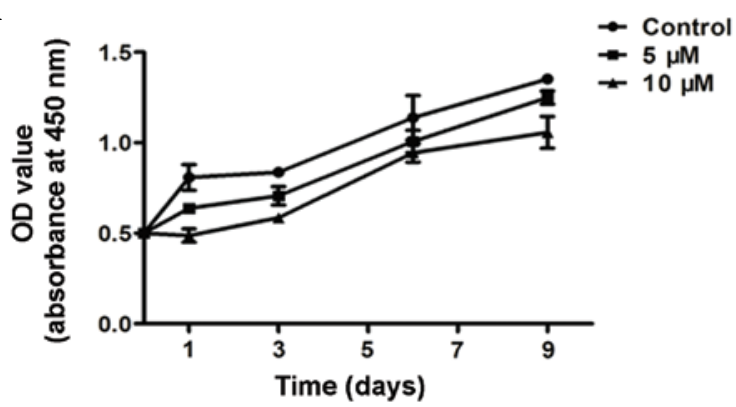

B

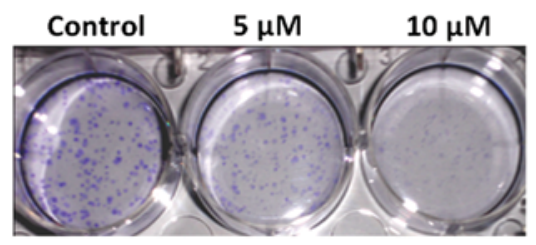

C

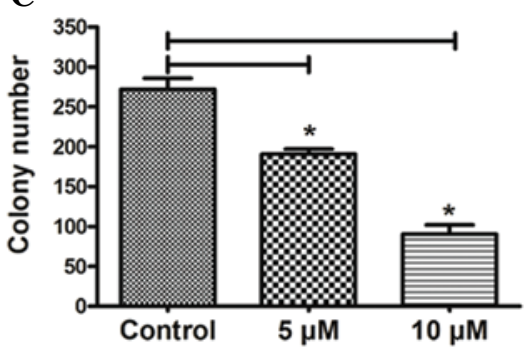

Figure 2. Decrease in proliferation and colony formation abilities of human chondrosarcoma cell line SW1353 following treatment with HPI-4. OD value (absorbance at $450 \mathrm{~nm}$ ) was used as an index to evaluate cell proliferation ability. (A) The first line chart shows that higher concentration of HPI-4 inhibited tumor cell proliferation with time (1,3,6 and 9 days). (B) HPI-4 clearly inhibited proliferation ability so that a single cell could not form a colony with a large range after treating with HPI-4 for 4 weeks and (C) the histogram displays the average number of colonies. The test groups were compared with the control group $\left({ }^{*} \mathrm{P}<0.05\right)$. 


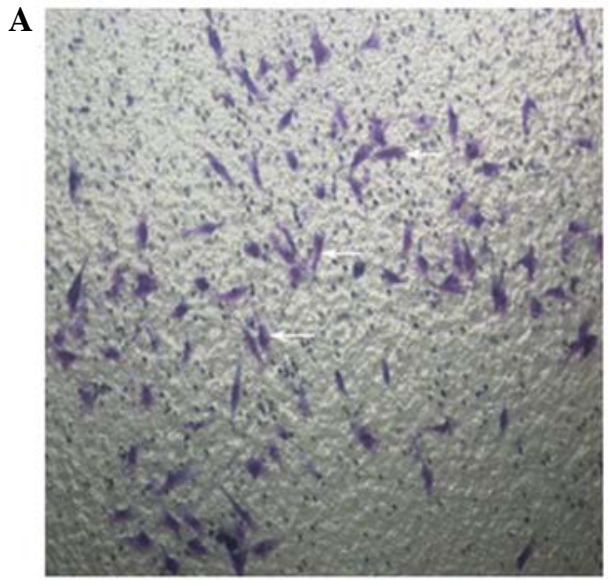

Control

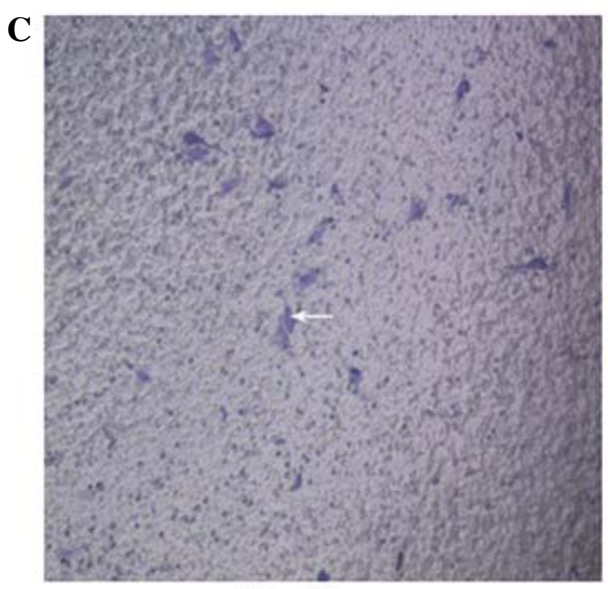

$10 \mu \mathrm{M}$

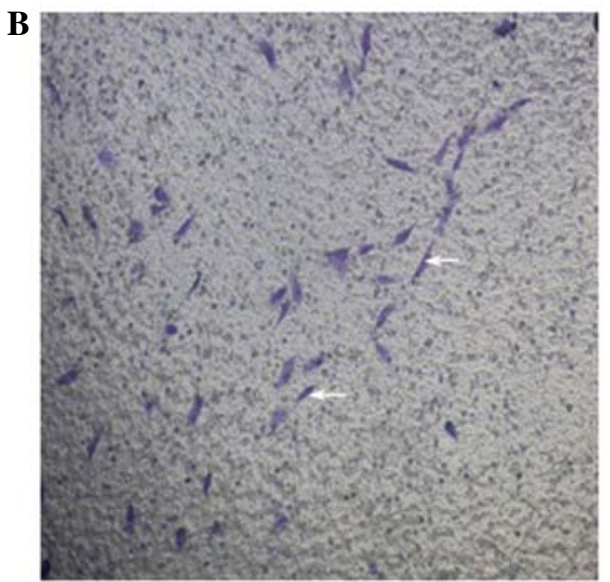

$5 \mu \mathrm{M}$

$\mathbf{D}$

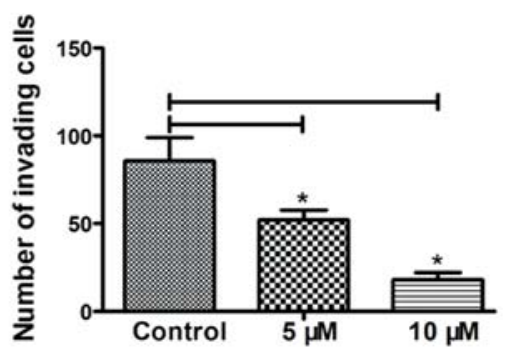

Figure 3. HPI-4 visibly suppresses chondrosarcoma cell invasion ability. Tumor cell invasion ability was assessed by Transwell assay. (A-C) Different concentrations of HPI-4 (0,5 and $10 \mu \mathrm{M})$ resulted in different numbers of penetrating cells to the lower surface (white arrows). (D) Cells were counted in 10 randomly selected microscopic fields and the histogram shows the average number of penetrating cells. The test groups were compared with the control group $\left({ }^{*} \mathrm{P}<0.05\right)$.

pathway, the MAPK/ERK pathway was also restrained by HPI-4, mainly through inhibiting ERK and phosphorylatedERK (p-ERK) proteins to downregulate downstream MMPs (Fig. 6A-D).

HPI-4 blocks the HH signaling pathway by disturbing ciliogenesis. We previously demonstrated that an apparent activation of HH signaling pathway exists, which may be associated with tumorigenesis in human chondrosarcoma (Fig. 1). The mechanism of HPI-4 as an inhibitor of HH signaling pathway is not fully clear, although some studies have demonstrated that this drug does not act on conventional Smo receptor (14). However, primary cilia are an indispensable part of the $\mathrm{HH}$ signaling pathway. This cell organelle can be counted and described under the field of vision and was regarded as an observed morphology symbol of cell cycle $(15,16)$. In order to detect whether HPI-4 realizes its function through interacting with primary cilia, we used fluorescence microscope to observe the expression percentage of primary cilia. Normal chondrosarcoma SW1353 cells present primary cilia $20.55 \pm 4.40 \%$, while adding $5 \mu \mathrm{M}$ HPI-4 resulted in a decrease to $15.06 \pm 5.15 \%$ $(\mathrm{P}<0.05)$ and $10 \mu \mathrm{M}$ to $7.38 \pm 1.91 \%(\mathrm{P}<0.001)$. The results confirmed that HPI-4 distinctly downregulates ciliogenesis, particularly at high concentrations (Fig. 5A-D). Moreover,
RT-PCR analysis showed that the expression of cilia microtubule transporters IFT88 significantly decreased $(\mathrm{P}<0.05)$ and the $\mathrm{HH}$ pathway target genes PTCH1 and GLI1 decreased distinctly $(\mathrm{P}<0.05)$, but only the higher concentration of HPI-4 suppressed Smo receptor expression. Thus, we speculated that HPI-4 may inhibit the action of the HH pathway by disrupting ciliogenesis rather than by suppressing Smo (Fig. 7E and F).

HPI-4 inhibits the expression of PTHrP regulated by reducing GLI2. HH and PTHrP play an important role in regulating the growth process of bone and cartilage. In normal cartilage tissue, an Ihh/PTHrP negative feedback loop exists. HH signaling pathways activate downstream GLI transcription factors to regulate the generation of PTHrP (2-6). We previously showed that GLI2 and PTHrP have a certain collaborative expression in the human chondrosarcoma tissues with apparent activation of the HH signaling pathway (Fig. 1E). As shown in Fig. 7, after stimulating chondrosarcoma cells with different concentrations of HPI-4, ciliogenesis-related microtubules transporter IFT88 was inhibited, which led to primary cilia dependency. HH pathway transduction was blocked and downregulated transcription factor protein GLI2. Thus, chained along with suppression of PTHrP protein and mRNA expression simultaneously (Fig. 7A-D). 
A

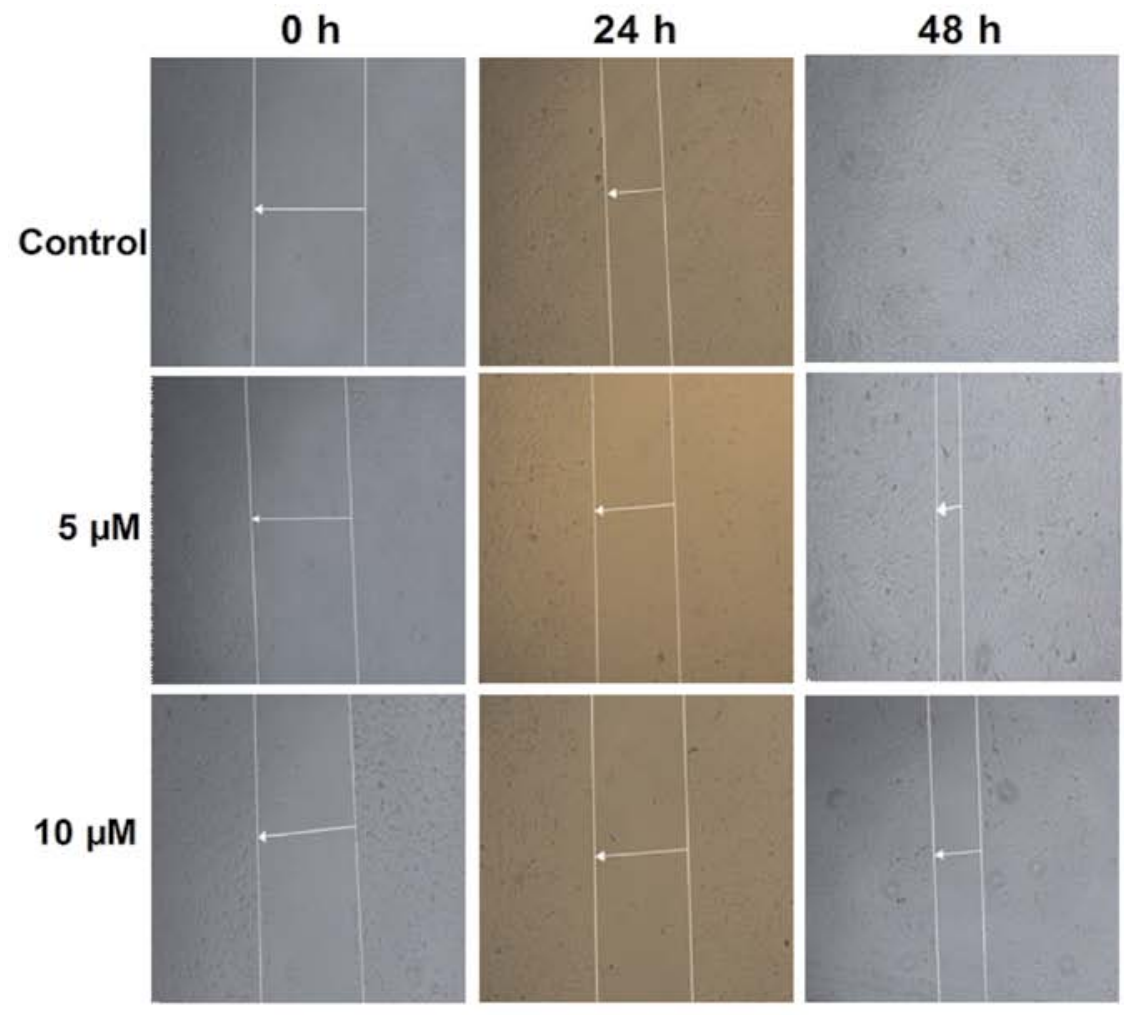

B

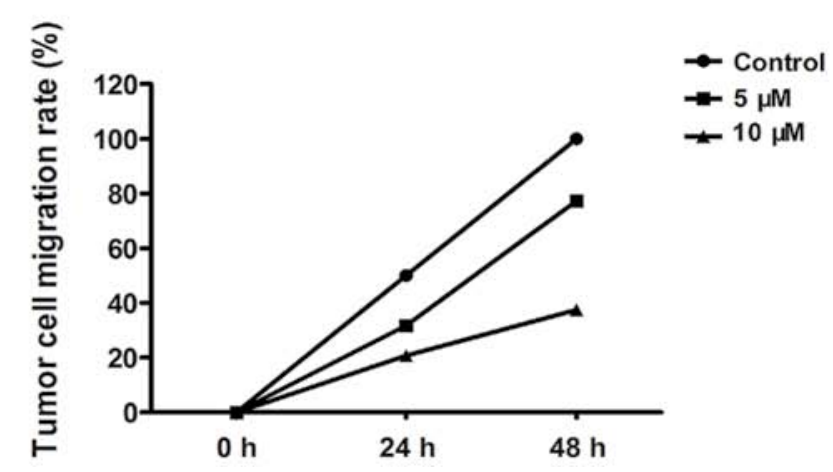

Figure 4. Tumor cell migration ability is downregulated by HPI-4. Wound closure assay was used to illustrate tumor cell migration ability. Confluent monolayer of SW1353 cells was wounded by scratching with a pipette tip. (A) Under different concentrations of HPI-4 treatment $(0,5$ and $10 \mu \mathrm{M})$, cell migration speed recorded visible differences at 24 and 48 h. (B) Line chart shows average migration rate of cells. HPI-4 clearly inhibited tumor cell migration ability and delayed wound healing time.

\section{Discussion}

As a type of malignant tumor secreting cartilage-like matrix, human chondrosarcoma has different general properties from other bone tumors. Depending on the tumor differentiation degree, the malignant severity is also different. Chondrosarcoma always occurs with a proclivity for long and flat bones, the main parts of weight bearing structures. This tumor can invade surrounding tissues and the consequences caused by mechanical structure damage cannot be ignored. Due to secreting cartilage-like matrix and lacking vascular distribution, chondrosarcoma is not sensitive to radiotherapy or chemotherapy and has high risks of recurrence and metastasis after surgical resection (1). Therefore, controlling the invasion and metastasis is key in guiding the treatment and improving prognosis of chondrosarcoma.
The HH signaling pathway is an important pathway in the progression of bone and cartilage development. Previous studies have confirmed the existence of HH/PTHrP negative feedback loop in normal growth plates to regulate cell growth and differentiation (1-3), but it is absent in cartilage neoplasia $(2,12)$. Furthermore, abnormal activation of HH signaling was found in different sources of malignant tumors, such as breast cancer, human renal cell carcinomas and gastrointestinal malignant tumor (7-11). Nevertheless, the specific pathogenesis for the abnormal activation of $\mathrm{HH}$ pathway that induces oncogenesis remains unclear. In the present study, we detected that apparent expression of protein Ihh, GLI1, GLI2 and PTHrP existed in human chondrosarcoma and this level of $\mathrm{HH}$ signaling activation was accompanied by strong proliferation ability. By using HH signaling pathway inhibitor HPI-4 to stimulate chondrosarcoma cell line SW1353 
A

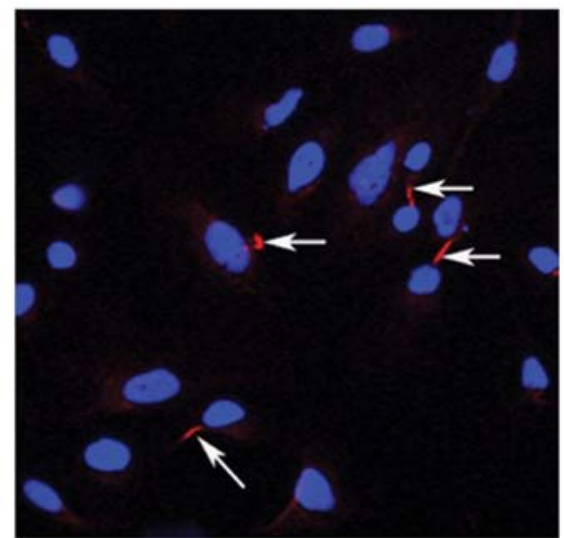

Control

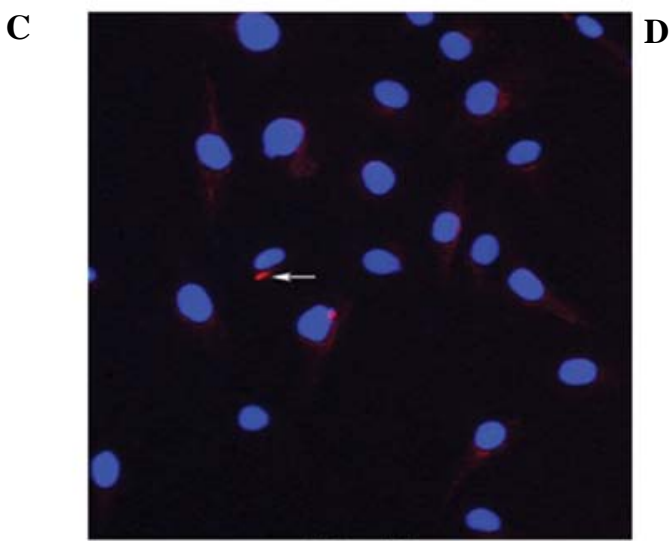

C

$10 \mu \mathrm{M}$

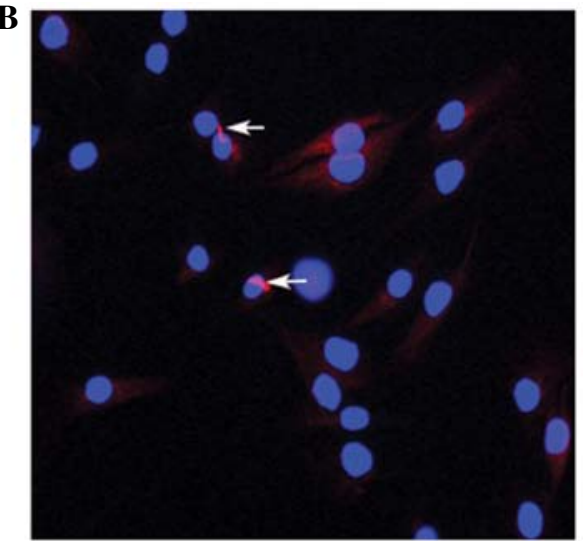

$5 \mu \mathrm{M}$

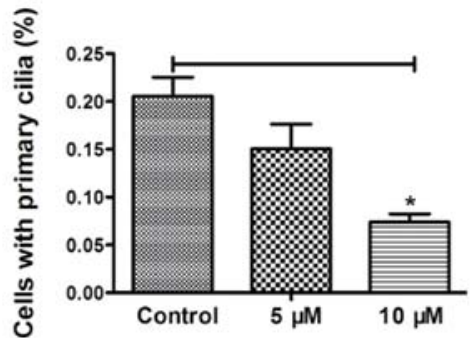

Figure 5. HPI-4 perturbs chondrosarcoma cell ciliogenesis. Human chondrosarcoma cells SW1353 were stained with acetylate- $\alpha$ tubulin (red) antibody to detect primary cilia. Comparison of the (A) control group and the (B and C) HPI-4-treated groups showed a lower manifestation of primary cilia (the white arrows) and (D) the histogram shows the percentage of cilia present. Cells were counted in 10 randomly selected microscopic fields and a P-value $<0.05$ was considered statistically significant compared with the control group $($ ( $\mathrm{P}<0.05)$.

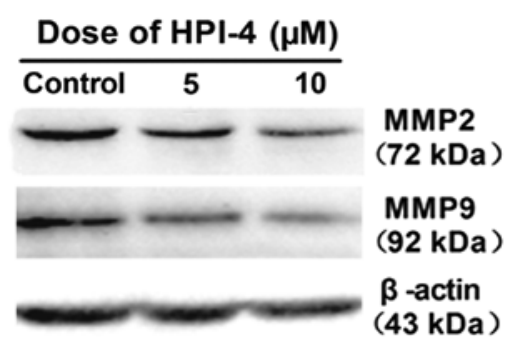

C

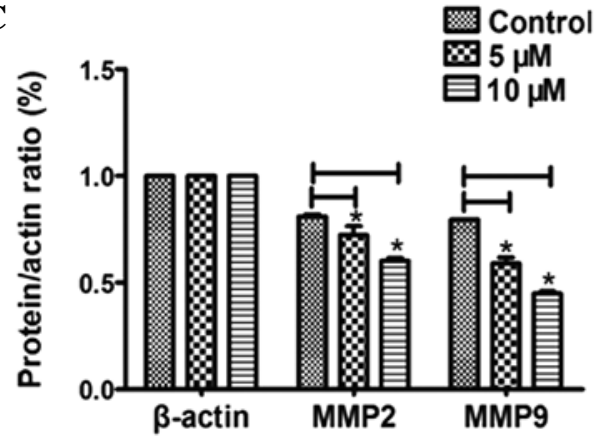

B

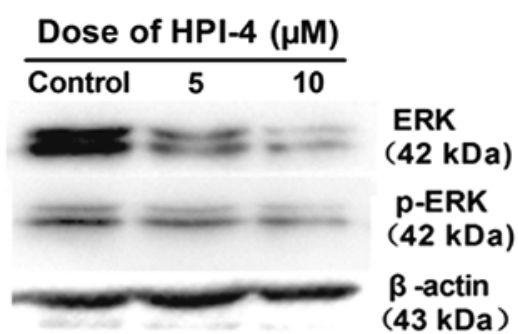

D

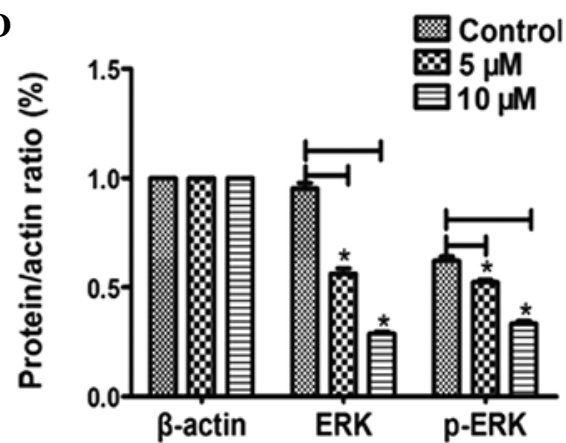

Figure 6. HPI-4 downregulates MAPK/ERK pathway-regulated downstream MMPs at the protein level. MMPs are regarded as a crucial index to assess tumor invasion ability and proved to be regulated by the MAPK/ERK pathway. (B and D) HPI-4 suppressed upstream key molecules ERK and the phosphorylated ERK, making (A and C) MMP2 and MMP9 decrease gradually with different concentrations of this inhibitor. A P-value $<0.05$ was considered statistically significant $(* \mathrm{P}<0.05)$. 
A

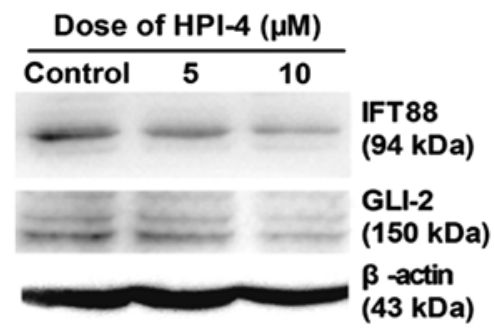

C
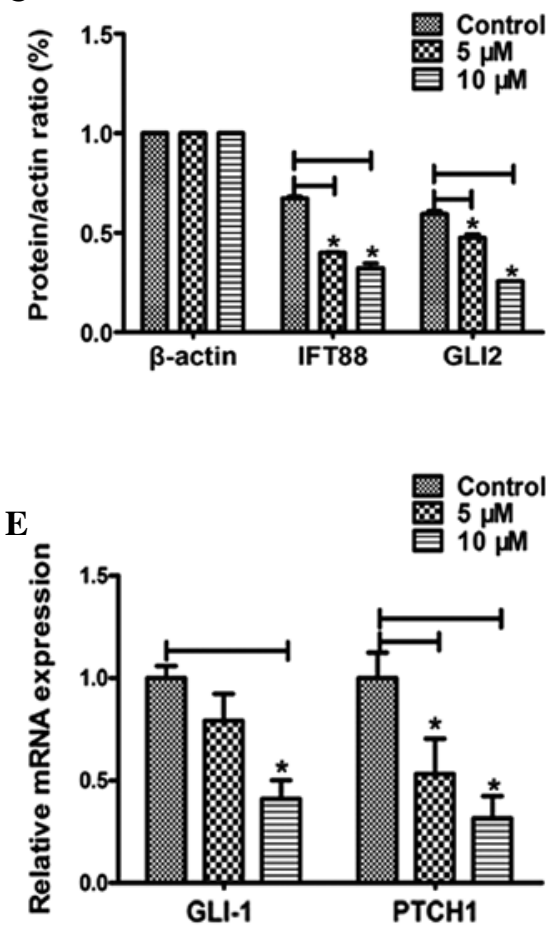

B

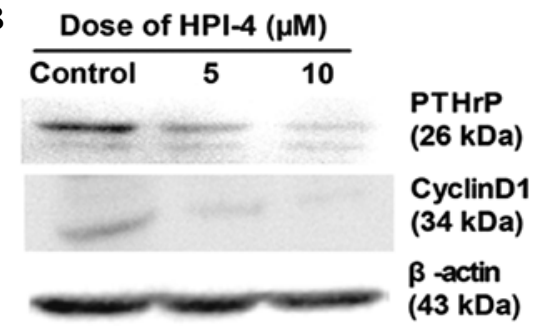

D

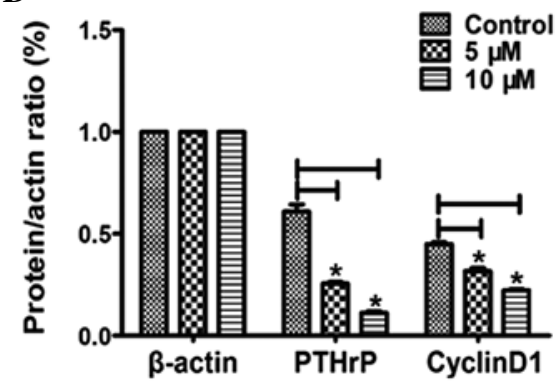

Figure 7. HPI-4 suppresses HH pathway-related signaling molecule expression at the gene and protein levels. (A-D) At the protein level, HPI-4 inhibited HH pathway cilia-related protein IFT88 and transcription factor protein GLI2 expression, then continuously downregulated target protein PTHrP which led to the decrease of cell cycle protein CyclinD1. (E and F) At the gene level, HPI-4 suppressed HH pathway target gene GLI1, PTCH1 expression and primary cilia-related gene IFT88, PTHrP. In addition, only a high concentration of HPI-4 (10 $\mu \mathrm{M})$ significantly inhibited Smo receptor gene expression. A P-value $<0.05$ was considered statistically significant $\left({ }^{*} \mathrm{P}<0.05\right)$.

in vitro, we illustrated that suppression of $\mathrm{HH}$ signaling could decrease tumor cell proliferation activation as time passed. Furthermore, MAPK/ERK signaling mediated tumor invasion-related proteins matrix metalloproteinases MMP2 and MMP9 $(22,23)$ expression declined visibly by use of $\mathrm{HH}$ signaling inhibitor HPI-4. This phenomenon indicates that there may exist a downstream crosstalk between $\mathrm{HH}$ signaling and MAPK/ERK signaling pathways. HH signaling inhibitor HPI-4 could have an obvious inhibitory effect on tumor invasion ability by regulating the expression of MMPs eventually. Therefore, we concluded that $\mathrm{HH}$ signaling pathway activation is closely linked with tumor cell proliferation activity and enhancing the decomposition of the extracellular matrix, thereby affecting tumor invasion and metastasis.

In the $\mathrm{HH}$ pathway inhibitor family, HPI-4 has a different interaction mechanism from other $\mathrm{HH}$ signaling pathway inhibitors. For example, the classical inhibitor cyclopamine is able to block Smo receptor and GANT51/GANT61 directly inhibits the expression of GLI family to interrupt HH pathway $(13,14)$, while HPI-4 may affect ciliogenesis to block this signaling pathway. Primary cilia are believed to regulate cell cycle progression and are considered a sign of ending cell division (15-18). In normal cells, primary cilia only present on non-proliferating cells and play an important role in initiating cilia-dependent $\mathrm{HH}$ pathway $(4,15-19)$. Cilia related IFT88 transfer in primary cilia and reflect the activity of material transportation in cilia (16). Theoretically, cilia microtubules disassembling during cell division progression and proliferation cells should lack primary cilia. However, after stimulating with HPI-4, the number of extracellular cilia decreased and intra-cilia transport protein IFT88 expressed in significant decline on gene and protein level accompanied by multiplication ability suppression. This illustrated that the drug might disrupt the normal function of cilia and inhibit cell proliferation simultaneously, accompanied by restraining the expression of HH downstream target genes GLI1 and PTCH1. Hence, we can infer that HPI-4 can downregulate $\mathrm{HH}$ activation by disrupting ciliogenesis and affecting their function. Primary cilia may be a crucial cell organelle involved in tumor initiation and progression. 
Regarded as a vital factor in the regulation of osteogenesis and osteolysis, PTHrP is closely related to $\mathrm{HH}$ signaling pathway $(5,6,20,21)$. Previous studies revealed that PTHrP expression could be increased by TGF- $\beta$ stimulation, which could upregulate $\mathrm{HH}$ downstream transcription molecule GLI2 expression. GLI2 could be activated and transferred into nucleus to launch a variety of cytokines transcription, especially PTHrP (6). By inhibiting the activity of the $\mathrm{HH}$ signaling, we found its downstream GLI2 expression decreased and endogenous PTHrP was restrained. As a result, we infer that the $\mathrm{HH}$ pathway inhibitor could affect to block osteolysis caused by PTHrP overexpression in tumor.

In summary, this study revealed that abnormal $\mathrm{HH}$ signaling pathway existed in human chondrosarcoma tissues. HPI-4, a new special antitumor compound, can perturb tumor cell ciliogenesis to block $\mathrm{HH}$ signaling pathway, consequently suppressing chondrosarcoma cell proliferation, invasion and migration abilities. These conclusions indicate that HPI-4 may be an effective therapy option to treat chondrosarcoma with abnormal activation of the $\mathrm{HH}$ signaling pathway.

\section{Acknowledgements}

This study was supported by the National Natural Science Foundation of China (Grant no. 81202121) and the Hubei Provincial Health Department of Young Scientists Fund (Grant no. QJX2012-05).

\section{References}

1. Björnsson J, McLeod RA, Unni KK, et al: Primary chondrosarcoma of long bones and limb girdles. Cancer 83: 2105-2119, 1998.

2. Tiet TD, Hopyan S, Nadesan P, et al: Constitutive hedgehog signaling in chondrosarcoma up-regulates tumor cell proliferation. Am J Pathol 168: 321-330, 2006.

3. Xu K, Guo F, Zhang S, et al: Blocking Ihh signaling pathway inhibits the proliferation and promotes the apoptosis of PSCs. J Huazhong Univ Sci Technolog Med Sci 29: 39-44, 2009.

4. Hassounah NB, Bunch TA and McDermott KM: Molecular pathways: the role of primary cilia in cancer progression and therapeutics with a focus on Hedgehog signaling. Clin Cancer Res 18: 2429-2435, 2012.

5. Johnson RW, Merkel AR, Danilin S, et al: 6-Thioguanine inhibition of parathyroid hormone-related protein expression is mediated by GLI2. Anticancer Res 31: 2705-2712, 2011.

6. Johnson RW, Nguyen MP, Padalecki SS, et al: TGF- $\beta$ promotion of Gli2-induced expression of parathyroid hormone-related protein, an important osteolytic factor in bone metastasis, is independent of canonical Hedgehog signaling. Cancer Res 71: 822-831, 2011.
7. Oue T, Uehara S, Yamanaka $\mathrm{H}$ et al: Hedgehog signal inhibitors suppress the invasion of human rhabdomyosarcoma cells. Pediatr Surg Int 29: 1153-1158, 2013.

8. Che J, Zhang FZ, Zhao CQ, et al: Cyclopamine is a novel Hedgehog signaling inhibitor with significant anti-proliferative, anti-invasive and anti-estrogenic potency in human breast cancer cells. Oncol Lett 5: 1417-1421, 2013.

9. Zhuang Z, Wang K, Cheng X, et al: LKB1 inhibits breast cancer partially through repressing the Hedgehog signaling pathway. PLoS One 8: e67431, 2013.

10. Basten SG, Willekers S, Vermaat JS, et al: Reduced cilia frequencies in human renal cell carcinomas versus neighboring parenchymal tissue. Cilia 2: 2, 2013.

11. Yan R, Peng X, Yuan X, et al: Suppression of growth and migration by blocking the Hedgehog signaling pathway in gastric cancer cells. Cell Oncol (Dordr) 36: 421-435, 2013.

12. Ho L, Ali SA, Al-Jazrawe M, et al: Primary cilia attenuate hedgehog signalling in neoplastic chondrocytes. Oncogene 32: 5388-5396, 2013.

13. Lauth M, Bergström A, Shimokawa T, et al: Inhibition of GLI-mediated transcription and tumor cell growth by small-molecule antagonists. Proc Natl Acad Sci USA 104: 8455-8460, 2007.

14. Wu VM, Chen SC, Arkin MR, et al: Small molecule inhibitors of Smoothened ciliary localization and ciliogenesis. Proc Natl Acad Sci USA 109: 13644-13649, 2012.

15. Goto H, Inoko A and Inagaki M: Cell cycle progression by the repression of primary cilia formation in proliferating cells. Cell Mol Life Sci 70: 3893-3905, 2013.

16. Irigoín F and Badano JL: Keeping the balance between proliferation and differentiation: the primary cilium. Curr Genomics 12: 285-297, 2011.

17. Proulx-Bonneau S and Annabi B: The primary cilium as a biomarker in the hypoxic adaptation of bone marrow-derived mesenchymal stromal cells: a role for the secreted frizzled-related proteins. Biomark Insights 6: 107-118, 2011.

18. McGlashan SR, Haycraft CJ, Jensen CG, et al: Articular cartilage and growth plate defects are associated with chondrocyte cytoskeletal abnormalities in $\mathrm{Tg} 737^{\text {orp }}$ mice lacking the primary cilia protein polaris. Matrix Biol 26: 234-246, 2007.

19. Rich DR and Clark AL: Chondrocyte primary cilia shorten in response to osmotic challenge and are sites for endocytosis. Osteoarthritis Cartilage 20: 923-930, 2012.

20. Das S, Tucker JA, Khullar S, et al: Hedgehog signaling in tumor cells facilitates osteoblast-enhanced osteolytic metastases. PLoS One 7: e34374, 2012.

21. Mak IW, Turcotte RE and Ghert M: Parathyroid hormone-related protein (PTHrP) modulates adhesion, migration and invasion in bone tumor cells. Bone 55: 198-207, 2013.

22. Bartsch JE, Staren ED and Appert HE: Matrix metalloproteinase expression in breast cancer. J Surg Res 110: 383-392, 2003.

23. Chung TW, Lee YC and Kim CH: Hepatitis B viral HBx induces matrix metalloproteinase-9 gene expression through activation of ERK and PI-3K/AKT pathways: involvement of invasive potential. FASEB J 18: 1123-1125, 2004. 